\title{
A survey of ab-initiocalculationsshows that segregation-induced grain boundary embrittlement is predicted by bond-breaking arguments
}

\author{
Michael A. Gibson and Christopher A. Schuh ${ }^{*}$ \\ Department of Materials Science and Engineering, Massachusetts Institute of \\ Technology, 77 Massachusetts Avenue, Cambridge MA 02139 USA \\ Contact Information: \\ Michael A Gibson: $\underline{m}$ gibson@,mit.edu, 617-258-5032 \\ Christopher A Schuh: schuh@mit.edu, 617-253-6901 * Corresponding Author
}

\begin{abstract}
The segregation of solute atoms to grain boundaries can have a large influence on the mechanical behavior of polycrystals, particularly in metallic alloys. An overview of 400 calculations of solute-induced changes in grain boundary cohesion from 81separate studies quantitatively demonstrates that the majority of the variation in solute-induced changes in boundary cohesion is explained by simple bond-breaking arguments. This trend is robust to changes in crystal structure and computational methods. The secondary contributions to embrittlement from other mechanisms, such as atomic size effects and charge transfer, are quantified and discussed as well.
\end{abstract}

Keywords: GrainBoundary Segregation; Embrittlement; Ab-InitioCalculation; Surface Segregation; Fracture

(C) 2015. This manuscript version is made available under the Elsevier user license http://www.elsevier.com/open-access/userlicense/1.0/ 
Grain boundaries (GBs) often mediate failures in engineering alloys, most commonly due to the segregation of impurities. GB embrittlement is usually associated with impurities' effects on GB cleavage, i.e. the separation of the boundary to form free surfaces. A seminal effort at understanding solutes' effects on GB cohesion was provided by Seah[1], who used a regular solution-based analysis to argue that boundary cohesion should be mostly determined by the intrinsic strength of the bonds formed by a solute relative to those of the base metal, as quantified by the difference in elemental sublimation enthalpies of the solute and solvent. However, at the time of Seah's work, the prediction of whether a solute would embrittle a boundary could only be validated against qualitative observations of decreases in toughness upon introduction of a given solute, and controlled studies were mostly limited to Fe-based systems.

Since Seah's original work, a large number of atomic-scale simulations and more detailed experiments have been performed across a variety of systems. These detailed analyses have lead to a wide variety of posited mechanisms for GB embrittlement, among them:

- oversize atoms induce local tensile strains which ease boundary decohesion (i.e. atomic size effects)[2],

- low-energy bonds across the interface permit facile surface formation [3, 4],

- formation of covalent bonds due to segregation affectsbonding betweenadjacent atoms [5, 6], and

- reductions in 'bond mobility' reduce the amount of possible plastic deformation at the GB [7-9]. 
Certainly all of these proposed mechanisms have relevance in specific systems, but quantitative mechanistic data is limited to a minority of studies [10-12].

It is relevant for alloy design to develop a global, more general understanding of solute-induced changes in GB cohesion. The large amount of quantitative computational data now available enables us to make such a broad, quantitative analysis of solutes' effects on grain boundary cohesion. In this Letter, weshow thatthe energetic analysis of Seah is quantitatively confirmed by such newer data. We also generalize his analysis to systems beyond Fe. This type of aggregative analysis is analogous to Ashby and coworkers' studies on diffusion [13] and fracture mechanisms[14-16], where the authors gathered data from a large number of studies to provide higher-level understanding of physical trends, despite the inherent differences among the systems studied.This type of analysis is distinct from the meta-analytical techniques common in the medical and social sciences; we do not in this paper aggregate inherently uncertain studies to infer the effect size of a single phenomenon. Rather, we gather a large set of studies on fundamentally distinct materials phenomena to demonstrate that they all are predicted with fairly simple underlying models, demonstrating a common basis for these phenomena.

We first reiterate and further develop Seah's original energetic analysis. In a view not restricted to the regular solution approximation, the change in a grain boundary's cohesive energy due to introduction of a solute, $\Delta E_{\mathrm{B}}$, can be approximated as a sum of effects due to bond-breaking, chemical interactions, and atomic size effects [17-19]:

$\Delta E_{\mathrm{B}} \cong \Delta E_{\text {Bond }}+\Delta E_{\text {Chem }}+\Delta E_{\text {Size }}$.

In the following paragraphs we estimate these three terms in turn to assess their relative importance. 
The bond-breaking term, $\Delta E_{\mathrm{B} o n d}$, represents the change in GB cohesive energy simply due to the replacement of solvent-solvent bonds with solute-solute ones at the boundary upon solute segregation. $\Delta E_{\text {Bond }}$ is estimated as thedifference in cohesive energies betweenthe solute and the solvent, $\Delta E_{\text {Bond }} \cong\left(E_{\text {solvent }}^{\text {coh }}-E_{\text {solute }}^{\text {coh }}\right) / 3[17]$, with the factor of three included because only a fraction of an atom's bonds are broken during creation of a surface. Fig. 1Ashows the distribution of elemental cohesive energies according to Kittel [20], ranging from close to zero for the noble gases to $8.9 \mathrm{eV} /$ atom for W. For typical engineering alloys, $\Delta E_{\text {Bond }}$ shouldusuallybe on the order of one eV/atom.

The $\Delta E_{\text {Chem }}$ term represents the effects of chemical interactions on the strength of bonding across the interface.One[21]can estimate the per-atom effects of chemical interactions between elements from the molar energiesof formation of their compounds[21,22],i.e., $\Delta E_{\text {Chem }}=1 / 3 \Delta E_{\text {Form }}$, the factor of three again accounts for the fraction of bonds lost upon decohesion. The distributions of formation energies in Fig. $1 \mathbf{B}[23,24]$ show that transition metal and transition metal-semimetal binaries typically exhibit $\Delta E_{\text {Form }}$ around $-0.2 \mathrm{eV} /$ atom, implying $\Delta E_{\text {Chem }} \cong-0.1 \mathrm{eV} /$ atom. Chemical effects can be larger for ionic and covalent solids (Fig. 1B) but for the present purposes the order of magnitude $\Delta E_{\text {Chem }} \cong 0.1 \mathrm{eV} /$ atom is a reasonable representation for most cases.

An upper bound on atomic size effectson boundary cohesion $\left(\Delta E_{\text {Size }}\right)$ isthe amount of elastic energy stored due to atomic size mismatch in the crystalline bulk; this energy would be at least partially released by segregation, which is what makes this an upper bound estimate for $\Delta E_{\text {Size }}$. An estimate of this energy according to continuum theory is [25]: 
$\Delta E_{\text {Size }} \leq \Delta E^{\text {elastic }}=\frac{24 \pi K_{\mathrm{B}} G_{\mathrm{A}} r_{\mathrm{A}} r_{\mathrm{B}}\left(r_{\mathrm{B}}-r_{\mathrm{A}}\right)^{2}}{3 K_{\mathrm{B}} r_{\mathrm{B}}+4 G_{\mathrm{A}} r_{\mathrm{A}}}$

where $G_{\mathrm{A}}$ represents the shear modulus of the solvent, $K_{\mathrm{B}}$ the bulk modulus of the solute, and $r_{\mathrm{A}}, r_{\mathrm{B}}$ the atomic radii of the solvent and solute, respectively.Fig. 1Cshows the distribution of $\Delta E_{\text {Elastic }}$ for alltransition metal solute-solvent pairs. The values center around $0.1 \mathrm{eV}$ and reach at most $2 \mathrm{eV}$. While some extremely large elements from outside the transition metal series may exhibit larger elastic effects, e.g.the rare earths, Fig. 1C and the inequality in Eq. 2 demonstrate that the vast majority of solute-solvent pairs, should exhibit $\Delta E_{\text {Size }} \leq 0.5 \mathrm{eV} /$ atom, and for most solutes $\Delta E_{\text {Size }} \leq 0.1 \mathrm{eV} /$ atom.

The three panels of Fig. 1 can be directly compared to the three terms of Eq. (1), and support the main hypothesis of Seah [1]: elastic and chemical effects in grain boundary embrittlement are expected to be smaller, second order effects when compared to the straightforward change in cohesive energy caused by the replacement of solvent atoms at the grain boundary. In other words,segregation-induced changes in boundary cohesion should be well-correlated with bond-breaking analogues.

In atomistic simulations, a solute's embrittling potency, $\Delta E_{\mathrm{B}}$, iscalculatedas the difference in the work of separation of agrain boundary upon segregation of a solute atom, B, to the boundary:

$\Delta E_{\mathrm{B}}=W_{\text {pure }}^{\text {sep }}-W_{\text {impure }}^{\text {sep }}$,

where $W_{\text {impure }}^{\text {sep }}$ and $W_{\text {pure }}^{\text {sep }}$ refer to the work of separation of the impure, segregated boundary and the pure boundary, respectively. A positive embrittling potency corresponds to a lower work of separation upon introduction of the impurity to the boundary, and thus to an embrittled boundary. 
Lejcek and Sobrecently examined the Seah hypothesis by comparing experimental and computationalembrittling potenciesin Fe to elemental sublimation enthalphies [26], achieving acorrelation that is supportive of the Seah hypothesis. However, almost half of the results discussed from Ref. [26]came from an analytical model of solutes' effects on GB cohesion by Geng et al.[17],rather than a set of ab initiocomputed embrittling potencies; the trend supporting the Seah hypothesis in Ref. [7] becomes much weaker when these data are removed from consideration. In what follows, we re-create the correlation suggested by Lejcek and Sob in Fe-based systems by gathering additional studies, and also extend the discussion to a much broader range of metals that have been studied by ab initio methods. While the bond-breaking arguments laid out above should be agnostic to the choice of base metal, this has not, to our knowledge, been tested before.

We gathered a large setof computed embrittling potencies from the open literatureand other sources, including reports to funding agencies and calculations reported in conferences from industry(a complete list of references and the data taken therefrom, as well as our data selection procedures, are collectedin the accompanying Data in Brief article). The vast majority ofthe data is from density functional theory (DFT) calculations from studies on grain boundary segregation in metals. In total, our survey included 81 individual studies or publications, including 400 investigations of solute segregation for 182 uniquesolute-solvent pairs. The data was collected based on segregation in the dilute limit, such that the embrittling potency of the solute is considered only at the most favorable site for grain boundary segregation, but under the assumption that the solute remains at the same site during fracture (i.e. that crack 
propagation is fast relative to diffusion). The embrittling potencies listed in this paper thus represent an upper bound to the embrittling potency of the solute under slower cracking conditions.[27-30]

Prior to discussion of the data, we first emphasize that scatter must be expected when aggregating the results of many studies, especially because they examine different grain boundaries from different crystal structures, and because the studies use different computational methods (e.g. exchange-correlation functionals, software implementations, reciprocal space sampling, etc.).To avoid overplotting and to more accurately summarize trends across studies, if multiple embrittling potencies have been calculated for the same solute/solvent pair, we then take the mean of all calculations for that solute/solvent pair. Each point displayed is thus a representation ofthe field's average estimate of the embrittling potency for a given solute in a given solvent.

The embrittling potencies for solutes at Fe GB's exhibit a strong, positive correlation with the difference in elemental cohesive energies of the elements, $\Delta E^{\text {cohesive }}=E_{\text {solvent }}^{\text {cohesive }}-E_{\text {solute }}^{\text {cohesive }}($ Fig $2 \mathbf{A})$. The elemental cohesive energies are used as an analogue for the difference in bond strengths as opposed to the enthalpies of sublimation proposed by Seahbecause DFTcalculations effectively take place at zero Kelvin. The correlation in Fig 2Ademonstrates that simple bond-breaking arguments are indeed able to describe the majority of the variation in embrittling potencies for the Febased systems studied in the literature. Further, the slope of approximately one third is consistent with the simple physical argument that approximately one third of bonds are broken during decohesion. This analysis successfully re-creates the relationship suggested by Lejcek and Sob [26], but benefits from reduced scatter due to our inclusion 
of additional studies, our removal of the analytical data as discussed above, and our use of averaging when multiple data points were available for a single system.

In Figs. 2 Band2 $\mathbf{C}$ we extend the analysisto solutes in $\mathrm{Ni}$ and $\mathrm{W}$, for which the embrittling potencies show a similar trend as those for Fe, albeit over a wider energy range because of the larger variety of solutes studied. All of the available calculations of embrittling potencies are aggregated and presented in Fig 3A. The positive correlation in Fig 3Asupportsthe Seah hypothesis, but a significant amount of scatter remains.A more meaningful comparison across solvents should consider the degree to which a solute changes the cohesion of a grain boundary relative to the cohesive energy scale of the solvent, giving a dimensionless embrittling potency,

$\Delta \varepsilon_{\mathrm{B}}=\Delta E_{\mathrm{B}} / E_{\text {solvent }}^{\text {cohesive }}=\left(W_{\text {impure }}^{\text {sep }}-W_{\text {pure }}^{\text {sep }}\right) / E_{\text {solvent }}^{\text {cohesive }}$

This amounts to a normalization of the ordinate axis in Fig 3A.A similar normalization provides the form for the abscissa: $\Delta \varepsilon^{\text {cohesive }}=\Delta E^{\text {cohesive }} / E_{\text {solvent }}^{\text {cohesive }}$.

The normalization presented in Fig. 3Buses the full set of data from Fig. 3A, and achieves a reasonable collapse of the many disparate studies into a single well-formed trend. The results for $\mathrm{Al}$ and $\mathrm{Mg}$, which were difficult to discern on the absolute scale of Fig $3 \mathbf{A}$, are now more pronounced on the normalized energy scale, while the trends for $\mathrm{Zr}$ and W, which appeared somewhat anomalous in Fig 3A, fall closer the trends for the other solvents once non-dimensionalized. Significantly, different solvents fall into different regions on the $\mathrm{x}$-axis of this plot: $\mathrm{Mg}$ has a rather low cohesive energy, and thus many alloying elements are expected to increase GB cohesion in Mg. In contrast, the high 
cohesive energy of W makes it fairly unlikely for alloying elements to significantly increase the cohesion of W GB's.

Non-dimensionalizing the embrittling potencies renders the data from different base metals ontocomparable scales, such that it is reasonable to use linear regression to summarize the data. Formally, the assumptions of independent observations and a constant variance inherent to linear regression are not satisfied in the present dataset, and so this analysis merely provides a one-number summary of the variation of the data, and an estimate of the slope. We find that the dimensionless difference in cohesive energies between the solute and the solvent is able to describe 59\% (adjusted $\mathrm{R}^{2}$ ) of the variation in dimensionless embrittling potencies via a one-parameter fit:

$\Delta \varepsilon_{\mathrm{B}}=\beta\left(\Delta E_{\text {solvent }}^{\text {cohesive }}-\Delta E_{\text {solute }}^{\text {cohesive }}\right) / \Delta E_{\text {solvent }}^{\text {cohesive }}$

with $\beta=0.41 \pm .05$. This slope is somewhat higher than the $1 / 3$ expected from simple physical arguments described earlier. This is possibly due to the covariance of other chemical and/or elastic effects with $\Delta \varepsilon_{\mathrm{B}}$, as discussed in greater detail below.

As we noted at the outset, aggregation of data from so many sources should be expected to lead to significant scatter due to, e.g., procedural differences. However, some of the deviations from the trend in Figs. 2 and 3likely reflect truephysics: the many systems presented exhibit differentchemical and elastic effects on decohesion. Following Fig. 1, such effects, while not dominant,should play a smaller but non-negligible role. Evidence for chemical and size effects as a source of scatter can be seen by comparing the various noble gas atoms as solutes in $\mathrm{Ni}$ (the right side of Fig 2B). These elements should behave identically in terms of their chemical interactions with $\mathrm{Ni}$, and also exhibit similar cohesive energies near zero. The variation in their embrittling potencies (which 
span a factor of two) should thus be mainly due to the scatter intrinsic to the data and the different atomic sizes of the noble gases.

Additional understanding of such second-order effects is gainedby examining those elements that fall well above the general trend. For example, the rare-earths, in the middle of Fig $2 \mathbf{B}$, and carbon, on the far left. For the rare-earth elements this is almost certainly due to their oversize radii relative to that of Ni leading to large amounts of elastically stored energy $\left(r_{\mathrm{RE}} / r_{\mathrm{Ni}}=1.39\right.$ to 1.55$)$ after segregation to the GB. For $\mathrm{C}$, the positive deviation is likely due to the anomalously negative cohesive energy of graphite as well as possible non-linearities due to unfavorable chemical interactions between $\mathrm{Ni}$ and $\mathrm{C} ; \mathrm{Ni}$ and $\mathrm{C}$ do not form carbides at equilibrium.

In summary, we find thatthe bond-breaking arguments made by Seah [1] 35 years ago for Fe-based systems are a general means to summarize the hundreds ofDFT computed changes in grain boundary cohesion due to solute segregation across alloy systems. Such data were not available to Seah, and previous studies have mostlyexamined small subsets of observations within a single base metal, such that global trends could not be convincingly revealed. Our aggregation of the data produced by the field to this point shows that when the data is considered in its totality and properly normalized, it convincingly conforms to the Seah analysis. What is more, these bond breaking trends are apparently robust to variations in crystal structure and computational methodology, with the majority of the variation in embrittling potencies explained based on just bondbreaking arguments.Deviations from these trends are due to a combination of chemical and atomic size effects, both of which present significant but generally small effects on 
embrittlement. Our findings, as well as the database presented in the online supplement, maybe useful in alloy design across metallic systems.

\section{Acknowledgments}

This work was supported by the US Army Research Office, under grant W911NF-14-10539. M.A.G. was further supported by the Department of Defense (DoD) through the National Defense Science \& Engineering Graduate Fellowship (NDSEG) Program. We thank A. Kalidindi and A. Lai for thoughtful discussions.

\section{References}

1. Seah, M.P., Adsorption-induced interface decohesion. Acta Metallurgica, 1980. 28(7): p. 955-962.

2. Schweinfest, R., A.T. Paxton, and M.W. Finnis, Bismuth embrittlement of copper is an atomic size effect. Nature, 2004. 432(7020): p. 1008-1011.

3. Gao, Q. and M. Widom, First-principles study of bismuth films at transitionmetal grain boundaries. Physical Review B, 2014. 90(14): p. 6.

4. Bauer, K.D., et al., A first principles investigation of zinc induced embrittlement at grain boundaries in bcc iron. Acta Materialia, 2015. 90: p. 69-76.

5. Messmer, R.P. and C.L. Briant, The role of chemical bonding in grain-boundary embrittlement. Acta Metallurgica, 1982. 30(2): p. 457-467.

6. Krasko, G.L. and G.B. Olson, Effect of boron, carbon, phosphorus, and sulfur on intergranular cohesion in iron. Solid State Communications, 1990. 76(3): p. 247-251.

7. Yuasa, M. and M. Mabuchi, Bond mobility mechanism in grain boundary embrittlement: First-principles tensile tests of Fe with a P-segregated Sigma 3 grain boundary. Physical Review B, 2010. 82(9): p. 5.

8. Haydock, R., The mobility of bonds at metal-surfaces. Journal of Physics CSolid State Physics, 1981. 14(26): p. 3807-3816. 
9. Goodwin, L., R.J. Needs, and V. Heine, A pseudopotential total energy study of impurity-promoted intergranular embrittlement. Journal of PhysicsCondensed Matter, 1990. 2(2): p. 351-365.

10. Janisch, R. and C. Elsasser, Segregated light elements at grain boundaries in niobium and molybdenum. Physical Review B, 2003. 67(22): p. 11.

11. Lozovoi, A.Y., A.T. Paxton, and M.W. Finnis, Structural and chemical embrittlement of grain boundaries by impurities: A general theory and firstprinciples calculations for copper. Physical Review B, 2006. 74(15): p. 13.

12. Lozovoi, A.Y. and A.T. Paxton, Boron in copper: A perfect misfit in the bulk and cohesion enhancer at a grain boundary. Physical Review B, 2008. 77(16): p. 14.

13. Brown, A.M. and M.F. Ashby, Correlations for diffusion constants. Acta Metallurgica, 1980. 28(8): p. 1085-1101.

14. Gandhi, C. and M.F. Ashby, Overview no. 5: Fracture-mechanism maps for materials which cleave: F.C.C., B.C.C. and H.C.P. metals and ceramics. Acta Metallurgica, 1979. 27(10): p. 1565-1602.

15. Ashby, M.F., C. Gandhi, and D.M.R. Taplin, Overview No. 3 Fracture-mechanism maps and their construction for f.c.c. metals and alloys. Acta Metallurgica, 1979. 27(5): p. 699-729.

16. Frost, H.J. and M.F. Ashby, Deformation-Mechanism Maps, The Plasticity and Creep of Metals and Ceramics. 1982: Pergamon Press.

17. Geng, W.T., A.J. Freeman, and G.B. Olson, Influence of alloying additions on grain boundary cohesion of transition metals: First-principles determination and its phenomenological extension. Physical Review B, 2001. 63(16): p. 9.

18. Murdoch, H.A. and C.A. Schuh, Estimation of grain boundary segregation enthalpy and its role in stable nanocrystalline alloy design. Journal of Materials Research, 2013. 28(16): p. 2154-2163.

19. Miedema, A.R., Surface segregation in alloys of transition-metals. Zeitschrift Fur Metallkunde, 1978. 69(7): p. 455-461.

20. Kittel, C., Introdcution to Solid State Physics. 8 ed. 2005, USA: John Wiley \& Sons.

21. Pauling, L., The Nature of the Chemical Bond. 3 ed. 1960, Ithaca, New York: Cornell University Press.

22. de Boer, F.R., et al., Cohesion in Metals: Transition Metal Alloys. Cohesion and Structure. 1989, New York, NY USA: Elsevier. 758.

23. Jain, A., et al., Formation enthalpies by mixing GGA and GGA plus $U$ calculations. Physical Review B, 2011. 84(4): p. 10.

24. Jain, A., et al., Commentary: The Materials Project: A materials genome approach to accelerating materials innovation. Apl Materials, 2013. 1(1): p. 11.

25. Friedel, J., Electronic structure of primary solid solutions in metals. Advances in Physics, 1954. 3(12): p. 446-507.

26. Lejcek, P. and M. Sob, An analysis of segregation-induced changes in grain boundary cohesion in bcc iron. Journal of Materials Science, 2014. 49(6): $p$. 2477-2482. 
27. Rice, J.R., Hydrogen and interfacial cohesion, in Effect of Hydrogen on Behavior of Materials, A.W. Thompson and I.M. Bernstein, Editors. 1976, Metallurgical Society of AIME. p. 455-466.

28. Hirth, J.P. and J.R. Rice, On the thermodynamics of adsorption at interfaces as it influences decohesion. Metallurgical Transactions a-Physical Metallurgy and Materials Science, 1980. 11(9): p. 1501-1511.

29. Hirth, J.P., Adsorption at grain-boundaries and its effect on decohesion. Philosophical Transactions of the Royal Society a-Mathematical Physical and Engineering Sciences, 1980. 295(1413): p. 139-149.

30. Rice, J.R. and J.S. Wang, Embrittlement of interfaces by solute segregation. Materials Science and Engineering a-Structural Materials Properties Microstructure and Processing, 1989. 107: p. 23-40. 


\section{Captions}

Figure 1: A logarithmic comparison of energy scales. A: Thenon-normalized probability distribution function, $\mathcal{P}$, of elemental cohesive energies B:Non-normalized

probabilitydistributions of the energies of formation , $-\Delta E_{\mathrm{Form}}$, fora representative set of transition metals with different classes of elements. C: The distribution of elastic mismatch energies for transition metal pairs. Data from Refs. [23, 24].

Figure 2: Embrittling potencies versus the difference in elemental cohesive energies for A Fe-, B Ni-, $\mathbf{C}$ and W-based systems

Figure 3: Summaries of embrittling potencies for all base metals. A Embrittling potencies versus the difference in elemenental cohesive energies. B Dimensionless embrittling potencies of solutes at grain boundaries versus dimensionless differences in cohesive energies of the solute and the solvent. Points are colored according to the solvent. 
Figure 1

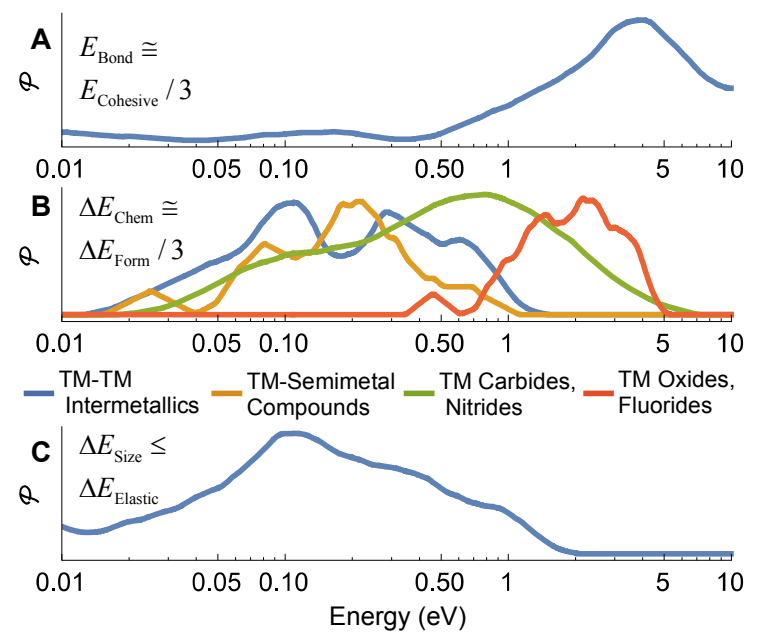


Figure 2

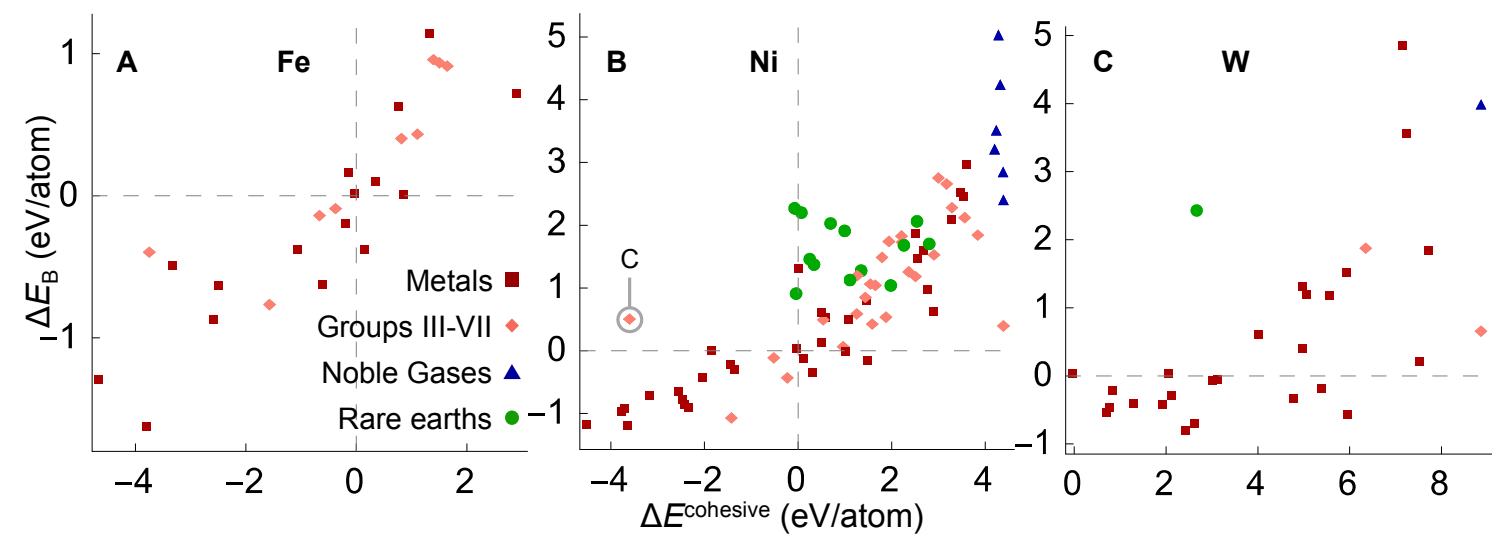


Figure 3
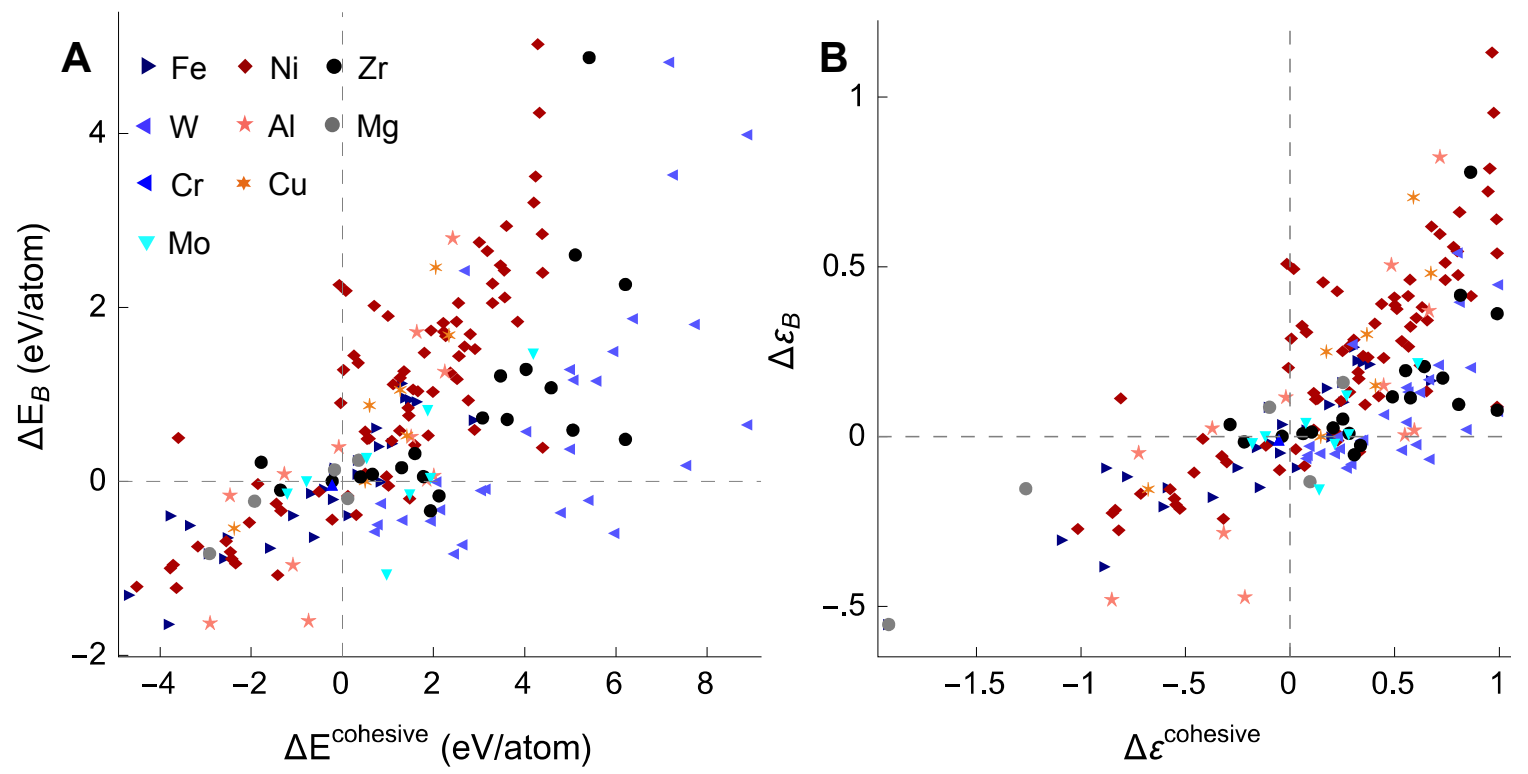


\section{*GraphífabiAhght5afts at a GB:}

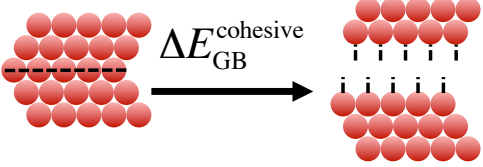

Is correlated to breaking bonds in general:

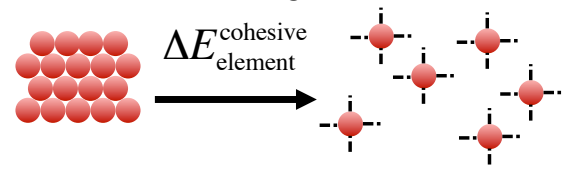

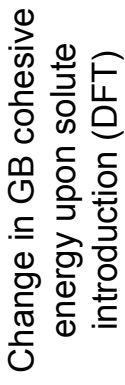

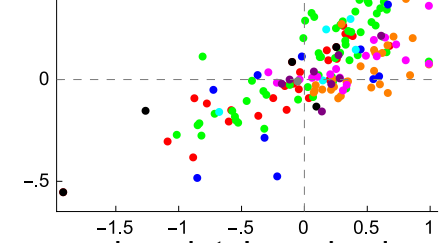

Difference in solute's and solvent's cohesive energies (tabulated) 